\title{
An Ontology to Automate Learning Scenarios? An Approach to its Knowledge Domain
}

\author{
Àngels Rius \\ Open University of Catalonia, \\ Barcelona, Spain
}

\author{
Miguel-Angel Sicilia, and \\ Elena García-Barriocanal \\ University of Alcalá, \\ Madrid, Spain
}

mriusg@uoc.edu

\author{
msicilia@uah.es, \\ elena.garciab@uah.es
}

\begin{abstract}
Automation or semi-automation of learning scenario specifications is one of the least explored subjects in the e-learning research area. There is a need for a catalogue of learning scenarios and a technique to facilitate automated retrieval of stored specifications. This requires constructing an ontology with this goal and is justified in this paper. This ontology must mainly support a specification technique for learning scenarios. This ontology should also be useful in the creation and validation of new scenarios as well as in the personalization of learning scenarios or their monitoring. Thus, after justifying the need for this ontology, a first approach of a possible knowledge domain is presented. An example of a concrete learning scenario illustrates some relevant concepts supported by this ontology in order to define the scenario in such a way that it could be easy to automate.
\end{abstract}

Keywords: ontology, knowledge domain, learning scenarios, automation.

\section{Introduction}

In recent years, the research by the eLearning community has been focused on topics such as standardization and learning objects. Nowadays, as a result of this research, we have a lot of standards and specifications developed. Examples are the LOM metadata standard (IEEE Learning Technology Standards Committee, 2002), which provides recommendations to annotate learning objects in order to facilitate their location and retrieval, the SCORM standard (Advanced Distributed Learning, 2004), which proposes guidelines to specify learning objects focusing on the content and package, the IMS LD specification (IMS Global Consortium, 2003b), which is centered on the description of the structure of learning units and the learning design process, and

Material published as part of this publication, either on-line or in print, is copyrighted by the Informing Science Institute. Permission to make digital or paper copy of part or all of these works for personal or classroom use is granted without fee provided that the copies are not made or distributed for profit or commercial advantage AND that copies 1) bear this notice in full and 2) give the full citation on the first page. It is permissible to abstract these works so long as credit is given. To copy in all other cases or to republish or to post on a server or to redistribute to lists requires specific permission and payment of a fee. Contact Publisher@InformingScience.org to request redistribution permission. the IMS DRI (IMS Global Consortium, 2003a) or the LORI (Simon, Massart, Van Assche, Ternier, \& Duval, 2005) specifications, which give recommendations to access in an interoperable way the repositories that allocate learning objects. It proves that the learning object topic has been the focus of the research from different points of view. However, if we want to construct a Learning Management System (LMS) based on these 
standards and specifications and make it more intelligent and adapted to the learning needs, we must go beyond the learning object concept and consider some other wider concept such as the learning scenario.

From an instructional point of view and related to the learning scenario concept, many people consider a scenario of the student only or an educational experience based on some learning strategies. Later, we will describe the learning scenario concept in more detail, but now we will sketch its meaning in order to avoid any confusion. The concept of a learning scenario in this paper must be understood as a model of an expected sequence of events to achieve a goal related within the LMS. Furthermore, the scenarios will let us describe the most usual functions of the LMS, such as those related to a student or a group of students (the assessment of a individual student in a specific activity, the itinerary proposed by the instructor to achieve a learning objective or the monitoring of learning) or to other more administrative or organizational ones that do not involve the student directly like the preparation of a course, the publication of a suitable learning activity, or the purchase of a learning object for a given learning need and so on.

There is some related research work about our view of the learning scenario. In Sicilia and Lytras, (2005), the concept of learning-scenario-type is presented as a generalisation of the learning scenario concept and the use of the learning objects metadata as an infrastructure to support the main functions of the LMS. A first approach to the specification of such scenarios promoting their automation was given in Sánchez-Alonso and Sicilia, (2005) and Sicilia, Garcia-Barriocanal, Sanchez, Rius, and Pages (2004) through setting up the semantic conformance profiles (SCP). Also, in Rius, Sicília, García-Barriocanal, and Macarro (2006), an implementation mechanism of learning scenarios was suggested using the combination of SCP-BPM-BPEL; although this technique permits obtaining an executable code, it does not guarantee correct behaviour of the system at execution time, because the learning scenario needs to be previously defined in a consistent and non-ambiguous way.

In order to achieve the automation of the most usual functions that occur in a LMS from well defined learning scenarios, first of all, we propose to create a catalogue of learning scenarios and then obtain, for each of them, its formal description based on a learning scenario ontology. Later, this formalisation of learning scenarios will be useful to achieve an executable code of the described scenarios. With respect to the catalogue, there is a proposal of cataloguing primitive learning scenarios in Rius, Sicilia, and Garcia-Barriocanal (2007), which must be extended in the near future. Now the next goal is the creation of the ontology to give support to the formalisation of learning scenarios to ensure the correct behaviour of the system.

In the eLearning field, some other authors have developed ontologies. For example in Amorím, Lama, Sánchez, Riera, and Vila (2006) an ontology is suggested to describe the semantic meaning of the IMS LD specification (IMS Global Consortium, 2003b), which means describing a learning process within a learning unit according to IMS LD specifications. On the other hand, other ontologies have been created to describe learning contents (Kabel, Wielinga, \& de Hoog, 1999), interactions between students and learning systems in collaborative environments (Ikeda, Hoppe, \& Mizoguchi, 1995), the learning tasks (Mizoguchi, Sinitsa, \& Ikeda, 1996) and the learning objectives and workgroups (Inaba et al., 2001) among others. Furthermore, in Barros, Verdejo, Read, and Mizoguchi, (2002) an ontology to describe the elements that take part in the learning scenarios of a collaborative environment is presented. This ontology has the aim of describing the interactions and dependences between the agents involved in a collaborative learning scenario.

Most of the ontologies mentioned above were created to describe the elements of learning scenarios from a structural point of view. The ontology we propose is focused on the specification of learning scenarios with the aim of automating the main LMS functions, meaning defining the be- 
haviour of all the elements involved in every scenario from a dynamic perspective. In this sense, it is like Amorím et al.'s (2006) ontology, which describes the process to create a learning unit using the learning design process. Both use the ontology to describe it from a functional point of view instead of a structural one.

The goal of this paper is two-fold. First of all, we would like to justify the need to construct an ontology to support the automated specifications of learning scenarios and then we would like to sketch out its knowledge domain. In order to achieve this double goal, the paper is structured as follows: firstly, an introduction; secondly, the justification of the proposed ontology; thirdly, the analysis of the scope of the ontology is stated; fourthly, the relevant concepts of its knowledge domain and an example of a scenario to illustrate how these concepts, taxonomies and rules can be used in the description of this specific scenario, and finally the conclusions and future work.

\section{Why do We Need an Ontology of Learning Scenarios?}

One of the most accepted definitions of an ontology in the field of computer science is the one given by Gruber (1993), who defines an ontology as "an explicit formal specification of a conceptualisation," understanding by conceptualisation an abstract and simplified vision of the world to be represented. Thus, an ontology permits the capturing of knowledge regarding a concrete domain. Although an ontology contributes to defining a conceptual scheme from reality, we do not have to think that this is like the conceptual scheme of an information system. An ontology not only represents taxonomy concepts and its interrelations, it also has instances. Furthermore, while ontologies usually represent static knowledge, they can also represent dynamic knowledge.

Independent of the ontology in question, ontologies bring about advantages both at compilation as well as at execution time (Conesa, 2008). On the one hand, during the development of an information system, the creation of an ontology can be useful in order to:

1. improve the consensus among the participants while the system is being created,

2. reuse the knowledge of the conceptual model in the activity model,

3. facilitate the comprehension of the knowledge domain both in terms of tasks and functions, and

4. validate previously created schemes.

On the other hand, in terms of execution time, an ontology brings about benefits that affect the effectiveness of the system: 1) improving the level of communication between different agents due to the support offered in the communication languages, which makes consensus between collectives possible, 2) integration of different data sources, 3) promoting the interoperability between different applications, 4) facilitating interpretation in natural language, 5) modelling the semantic content of web pages, and 6) supporting e-commerce applications.

If we consider a concrete ontology based on learning scenarios, with the aim of supporting the automation or semi-automation of learning scenarios and adding to it some general benefits, the ontology proposed will be especially successful for many reasons:

1) It will constitute a framework to describe learning scenarios formally, so it will guarantee a consistent and not an ambiguous behaviour of the LMS.

2) Its creation will lead us to define its knowledge domain, so it will be possible to obtain a deep knowledge of the scenario concept and other related concepts as well as the identification of all the required elements for its specification. 
3) The sharing of the same information structure among people and agents promotes knowledge reusing.

4) The creation and instantiation of the ontology will permit the creating of new learning scenarios and it will help us to construct a catalogue of learning scenarios, which does not exist at this time.

5) The separation between the knowledge domain and the base of knowledge will facilitate the obtaining of a detailed analysis of the knowledge domain, and it will be useful to develop applications that use the same set of data and the same information structure represented by the ontology.

As we are interested in the achievement of an ontology oriented to the automation (full or partially) of learning scenarios, it will be very important that such an ontology is going to capture all the details related to the specification of learning scenarios, including the behaviour of the LMS, repositories, and other systems that will take part in the different scenarios. This ontology will permit, the categorising of the high level concepts, which means the concepts related to the ontological structure. This ontology will also allow us to categorise the concepts related to dynamic issues of learning scenarios. As this ontology will need to model learning scenarios taking into account the behaviour of the LMS at execution time, axioms will be used to establish semantic restrictions associated to the taxonomy of concepts. Thus, we consider the importance of constructing this ontology since it will help to obtain formal specifications of learning scenarios that can be translated to any executable specification language and so it will lead us to the achieved automation.

\section{Analyzing the Scope of the Ontology}

In order to create an ontology to formalise and automate learning scenarios, the first step consists of analysing the scope of the ontology to determine its knowledge domain. Thus, according to Noy and McGuinness (2001), it will be useful to provide a response to the following questions:

- Why are we going to use this ontology?

- What kind of queries must the ontology give a response to?

- Who is going to use and maintain the ontology?

\section{Why are We going to Use this Ontology?}

Although it has been mentioned in the previous section, we are now going to specify its possible uses:

1) To obtain formal specifications of learning scenarios oriented to their automation

It implies that the ontology may permit obtaining specifications oriented to the definition of executable processes, this means in terms of pre-conditions, post-conditions and restrictions. Furthermore, this specification must be mapped onto any executable process specification language to guarantee its implementation.

2) To catalogue learning scenarios

Creating a catalogue of learning scenarios implies the instantiation of the ontology. Adding a new instance is adding a new learning scenario. The ontology will define the taxonomy of scenarios in order to catalogue them. We propose at least to distinguish between essential scenarios and composed ones. Regarding elemental learning scenarios, a classification has 
been suggested according to its main function in order to facilitate the composition of new complex scenarios from other simpler learning scenarios by means of a set of rules.

3) To validate the behaviour of new scenarios

Adding new scenarios to the catalogue will require a previous validation. This validation must take into account the behaviour of the LMS and other related systems. Thus, the ontology will be useful to verify the consistent and not ambiguous behaviour of the learning system and other agents involved in scenarios to be defined, so it will avoid the generation of any type of conflicts.

4) To personalise learning scenarios

Once we have defined learning scenarios formally, each instance of a learning scenario can be updated taking into account the user profile, the contents or resources, the format presentation, and so on. This means that all the information generated during prior learning processes where the learner has been involved can be used to guide the learner in subsequent learning scenarios where he/she participates, with the aim of personalising them.

5) To monitor the behaviour of the learning scenarios within the LMS

The ontology can also be used to trace and control typical learning scenarios within the LMS. The information provided from this experience would be very useful for collecting the learner's behaviour in the learner processes where he/she has participated and to improve the functionalities of the LMS according to the real needs. Thus, this extra and detailed information may be used to provide support in the assessment of quality too.

\section{What Kind of Queries must the Ontology give a Response to?}

This question can be answered with a list of queries such as:

- What is a scenario?

- What elements are part of a scenario?

- Which is the goal of a given scenario?

- What kinds of scenarios do exist?

- Is there any scenario with a concrete purpose?

- What are the initial conditions that make it possible to carry out a concrete scenario?

- Which are the restrictions of a given scenario?

- How must a learning scenario be specified?

- What is the flow of information generated by a scenario during its execution?

- Who are the participants or actors involved in a learning scenario?

- What are the roles associated with each actor involved in a concrete scenario?

- How is the communication between the participants of a learning scenario going to be carried out?

- What are the processes that make the scenario possible?

- How must the processes be joined in order to achieve the final goal of the scenario?

- What is the execution order of the different processes involved in a concrete scenario? 
- What is the information needed by a process to initiate its execution?

- What information is returned by a process once it has finished its execution?

- What are the activities carried out by a process?

- What is the goal of each activity?

- What is the sequence of activities that constitutes a process?

- What information is needed in order to carry out an activity?

- What kind of information is generated during the execution of an activity?

- When does an activity finish?

- When does a process finish?

- When does a scenario finish?

- What are the different ways to finish a concrete scenario?

\section{Who is going to Use and Maintain the Ontology?}

First of all, we know this ontology is going to be used to support a specification technique of learning scenarios in order to define the consistent and not the ambiguous behaviour of learning processes. Thus, it will be used by anyone who would like to automate (full or partially) scenarios in learning technology.

This ontology is also going to be used by anyone who wants to create a catalogue of learning scenarios. Anyone who wants to validate existing learning scenarios will be able to use the ontology too. The learning scenario to be validated must be described in terms of the ontology, so it would be possible to check the correct functioning of the system while the scenario takes place. Furthermore, someone who requires updating any part of a scenario in learning technology will have to maintain the catalogue assuring its consistency. We have to note that the validation and the maintenance of the catalogue are applications of the ontology at compilation time.

Other possible uses of the ontology are related to execution time. For example, the ontology can be used to trace the LMS behaviour, so it means that according to the different states achieved during the execution of a scenario, the next action to be carried out or the scenario to be executed will be decided. It will also allow extending the LMS capabilities in the sense that this traceability will be used to personalise learning scenarios at different levels (contents or resources, format presentation, user profiles).

All the questions previously formulated up to now and its responses will help us to achieve a deeper comprehension of these concepts supported by the ontology. Therefore, they are going to be included in its knowledge domain, as will be outlined in the next section.

\section{The Knowledge Domain of the Ontology}

An ontology to support automated or semi-automated scenarios in learning technology will have as its kernel the learning scenario concept, and more specifically, the concept of specification of learning scenarios.

There is not a single definition for scenario as it has been used in several areas of different disciplines. Usually learning scenarios are understood as examples of classroom activities that reflect standard-based reform strategies and proficiency-based world language instruction (Tood et al., 1999), but they can be also defined as flexible tools for the design of systems that have no single 
form or way to use it (Toffolon, 2006). From this last perspective, a scenario establishes a link between the LMS functionality and the learning processes, between architecture and implementation components, and provides an anchor for change traceability. We agree with this last view of scenario, and we are going to describe its related concepts in order to classify them in taxonomies and define the structural part of the ontology according to it.

Every scenario describes a typical situation within the LMS with a specific goal. It has a name, its parameters, and its goal. The achievement of such a goal implies the participation of one or more entities in one or more processes. Thus, at first glance, a scenario can be seen as a set of processes and a set of entities in communication with each other in order to achieve the final goal. Moving to processes and entities, Table 1 shows an example of a specific scenario showing its basic properties.

Table 1: Basic properties of the entity Scenario, Acquisition of a LO scenario

\begin{tabular}{|l|l|}
\hline Name of the scenario: & Acquisition of a Learning Object \\
\hline Parameters of the scenario: & $\begin{array}{l}\text { Learning objective } \\
\text { Pedagogical requirements } \\
\text { Buying conditions }\end{array}$ \\
\hline Goal of the scenario: & $\begin{array}{l}\text { To purchase a LO according to a given learning objective, some pedagogical } \\
\text { requirements and some buying conditions. }\end{array}$ \\
\hline
\end{tabular}

Entities are the elements that participate in the scenario. There are different kinds of entities, but generally, we can distinguish between human entities, agent software entities, and resource entities. Usually the human entities are called actors and in the e-learning context they are used to act as a learner, an instructor, or an evaluator among others. The agent software entities are other systems that interacts with the LMS; for example a learning content management system or /and authoring tool could be some examples. Finally, the third kind of entities are the resources, which are always used by processes; a typical example could be the repository. In the example of the scenario presented in Table 1, the actors involved are: the Instructor (human entity) requesting the acquisition of a LO to the LMS, the repository (resource entity) that allocate the learning objects, the vendor system (agent entity), and the LMS (agent entity).

Processes are the procedures that permit change to the current state of the system. They carry out activities that usually imply the interaction between two or more actors. Each process has a main function, and it can be divided into sub-processes depending on their complexity. The subprocesses may be considered as smaller units of processes with a simpler goal. In the example of scenario presented in Table 1, the main processes identified are: 1) the search of the LO, 2) the selection of the most suitable learning object, and 3) the purchase of the LO. In this case we have not divided processes into subprocesses due to their simplicity.

The way actors take part in a scenario and how they establish communication between them are relevant issues in the configuration of any scenario. With reference to the way participants take part in a scenario, it is important to note that depending on the activity to be carried out they play one role or another. Then, the same actor can play different roles in the same scenario. In the example of the scenario presented in Table 1, the LMS plays different roles: 1) the LMS is searching the LO, 2) the LMS is selecting the most suitable LO, and 3) the LMS is purchasing the LO. The role concept must be understood as a pair entity-activity which means the actor playing the activity in question. Thus, in our example, the first role is derived from the fact that the LMS is searching the LO (LMS/entity- search of a LO/activity), the second role corresponds to the fact that the LMS is selecting the most suitable LO (LMS/entity- selection of the most suitable LO/activity), and the last role, the LMS purchasing the LO is derived from the pair (LMS/entitypurchase of the LO/activity). From this perspective the scenario can be seen as a sequence of 
roles oriented to the achievement of a given goal. In our example, the Acquisition of the LO scenario, the scenario is constructed by a sequence the three above mentioned roles.

The communication between the different entities involved in a learning scenario is another essential issue in the description of a learning scenario as we mentioned before. Actors that participate in a scenario need to establish communication between them in order to carry out their assigned roles. As the eLearning environment is a distributed eLearning environment, the event concept is very important. Events trigger processes and processes are constituted of sequences of roles, so events also trigger roles. Each role is activated or triggered by an entry event and, in order to activate the next role, it produces an exit event when it finishes. Hence, every specific learning scenario can also be seen as a sequence of pairs of events: as a flow of events that is time-dependant on the system's behaviour. The flow of events required to perform the scenario referred to in Table 1 is presented in Figure 1, as well as the participation of each entity in each process. The events generated by the LMS that activate the next role within the scenario are shown in the columns (Request_LO_acquisition, Request_selection_LO, Request_purchase_LO), each of them activating one of the processes to be carried out by the scenario. Some of these processes also need the collaboration of other entities in order to achieve their goals, and then other kinds of events are required, for requesting services. In the Acquisition of the LO scenario the interaction with the repository to search the LO that satisfy the requirements is an example and the interaction with the vendor system to carry out the economic transaction is another one.

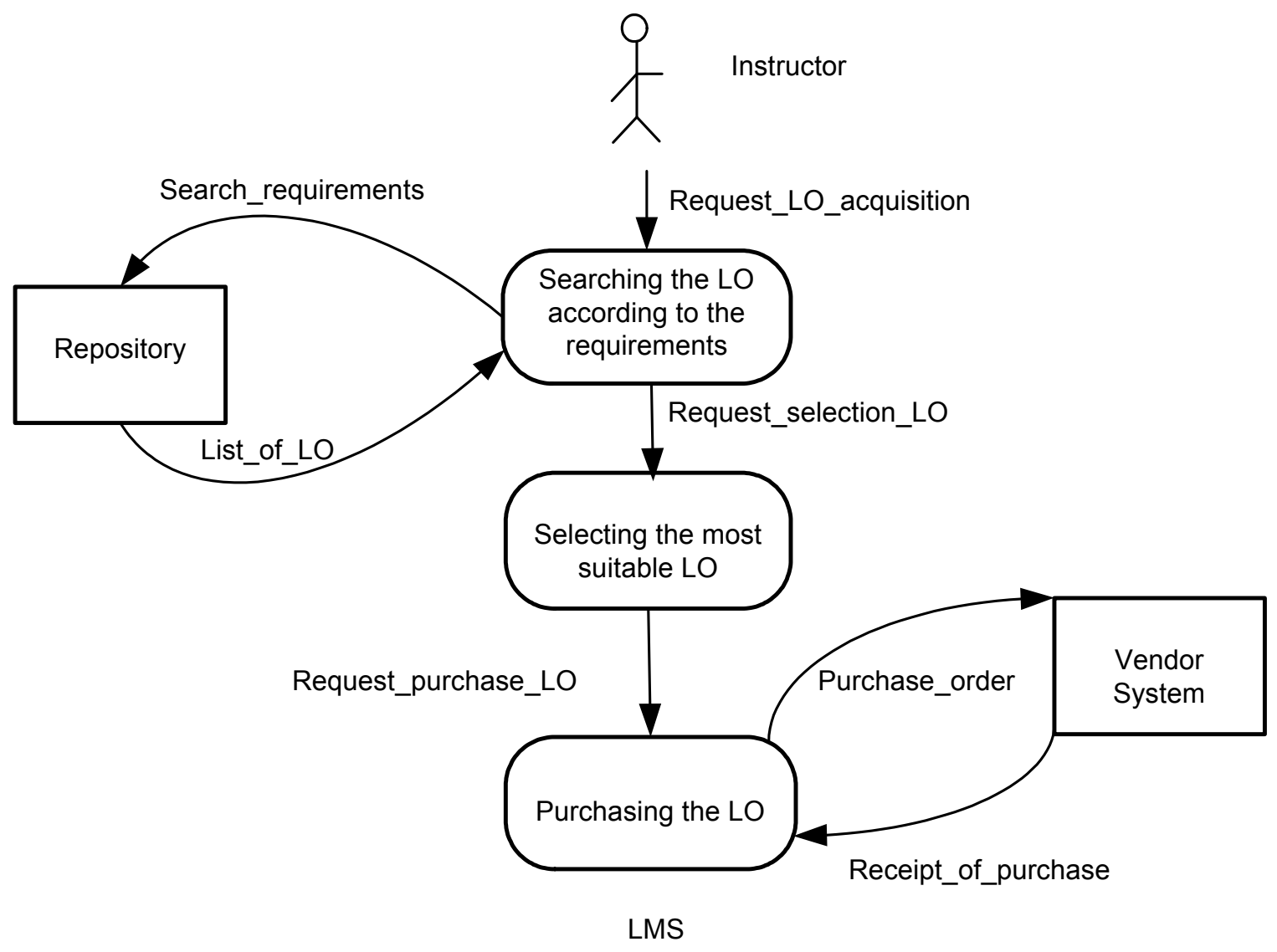

Figure 1: Diagram of the Acquisition of a Learning Object (LO) scenario.

Up to now, we have seen the main elements that configure a scenario and the two different perspectives of the learning scenario. One vision of a learning scenario is referring to the sequence of 
roles and the other, more dynamic than this one, is referring to the flow of events. Therefore, in our ontological structure we will define at least three taxonomies to classify entities, processes, and events. The role concept will be derived from entities and process concepts.

Apart from the three main taxonomies mentioned, a taxonomy of scenarios will be needed in order to classify them. In Rius, Sicilia, and Garcia-Barriocanal (2007), a primitive catalogue of learning scenarios based on some related standards and specifications is presented. It classifies the learning scenarios according to their complexity in primitive scenarios (those that can not be divided more) and complex scenarios. The primitive scenarios in turn can also be categorized into four depending on their goals: 1) scenarios related to the transfer of information through the LMS among actor and agent software entities; 2) scenarios focused on the preparation of information in order to be comprehended by the target (entity or process), 3) scenarios centred in management of resource entities, which includes the main functions to manage repositories and 4) scenarios that are used as support functions based on learning objects and learning processes. These primitive scenarios can be combined to produce new complex scenarios and these, at the same time, can be part of other more complex learning scenarios. For the composition of scenarios, we propose the use of rules to define them; the ontological structure is not enough, because its definition involves behaviour that depends on the execution-time.

Returning to the Acquisition of the LO example (Figure 1), the primitive scenarios that conform the scenario are the following: 1) the search of the LO, which is constructed by a sequence of two primitive scenarios: one for the preparation of a query (second kind of the primitive scenario taxonomy) and another for the search in the repository (third kind of the primitive scenario taxonomy); 2) the selection of a LO, which implies a request to an external service, so using the sendreceive primitive scenario (first group in the taxonomy); and 3) the purchase of LO also requesting the vendor system services (first group in the taxonomy) and the auditory of the purchase.

The domain of knowledge in which we are interested is specified by an ontology especially created to support the specification of learning scenarios, meaning obtaining formal specifications of processes that can be used to guide the learning scenario implementation assuring they are defined in a correct format and not in an ambiguous way. Hence, we propose defining the specification of scenarios in terms of pre-conditions, restrictions, and post-conditions. This kind of information will be expressed in terms of predicates. The precondition predicate will define the initial condition that leads us to the goal expressed by its condition, nd the restriction predicates will be some conditions to be checked during the execution of the scenario in order to guarantee the correct behaviour of the system. From this perspective, the composition of scenarios has to be seen as the alignment of preconditions and postconditions and so they must take into account the rules that define the composition.

At this point, we have mentioned the key concepts of a generic ontology. But if we want to use this ontology in the Open University of Catalonia (UOC) environment, we must restrict such scenarios to define UOC scenarios. In order to do it, we also need to increase the knowledge domain of the proposed ontology, because we need to define the scenario-templates of the UOC to adapt to the specific reality. These UOC scenarios will need to take into account the internal procedures of the organisation, its established rules, and so on. Furthermore, at a lower level, the scenarios will need to be defined by the tasks carried out in the scenarios, as can be the search of resources or the schedule of activities. With this purpose, we propose to incorporate the OKI-OSID specification (Open Knowledge Initiative, 2002), because it provides a wide set of open and extensible definitions of services for learning technology, it is specially targeted to the needs of the higher education community, and its definition interfaces are general in nature so that they can be incorporated in our ontology. It is also be possible to promote reusability by dividing the proposed ontology in several of them, but this will be decided during the creation of such ontology. 
Next, in order to clarify the concepts of the knowledge domain exposed above, let us move on to a more complete example.

\section{An Example of a Scenario: The Preparation of a Learning Activity in the UOC Environment}

The scenario proposed as an example is the preparation of a learning activity in the context of the UOC. It has the goal to create a new learning activity to assess the achievement of some learning objectives. While the learning activity is being prepared, some templates will be used in order to determine its structure or distribute tasks to create it. The result of this scenario is the learning activity saved in a repository to be released on a given date.

The description of this learning scenario will be presented identifying the main elements that conform such a scenario: the entities involved, the processes to be carried out, and the sequence of events that lead to the expected objective.

There are three participants involved in the scenario: 1$)$ the lecturer responsible for the subject (once the scenario is automated, the LMS will do his/her role), 2) the consultants (three in this example), and 3) the repository. The lecturer is a member of the UOC who has the maximum responsibility for the subject matter and coordinates the consultants who are the collaborative staff, who are in contact with the students enrolled in the course. The repository is a resource from which to retrieve the templates and to save the learning activities. The two first entities are actors and the third one is a resource.

The preparation of a learning activity requires the execution of three processes in sequence. First, the structure of the learning activity and its learning objectives must be known by searching the template of such an activity and consequently, to access the repository to retrieve this information. Secondly, once we know the kind of exercises to prepare, the distribution of such exercises among consultants, the role they must play and the dates to return them, the task to be performed by each of them is the next step. The different roles, tasks, and dates are determined according to the procedure of distribution of tasks established by the lecturer, which are specified in another template. Thus, this second main task also implies access to the repository and exchange of documents between consultants and the lecturer. Thirdly, the definitive learning activity is composed from the exercises previously developed using the structure template. Finally, this new learning activity is saved in the repository until the day of publishing.

The first main task is triggered by the request of the preparation of a learning activity, which in turn will activate other events that will initiate the other main processes previously mentioned, leading it to the new learning activity to be saved in a repository. The description of this specific scenario is illustrated in Figure 2.

In Figure 2, the preparation of a learning activity scenario can be explained in terms of the main concepts of the ontology. The edges represent the events and the direction of them indicates the flow of every pair of events that determines the role to be executed. Therefore, this scenario can be seen as a sequence of events (request_LA_event, request_distribution_tasks_event, request_gathering_tasks_event, return_LA__event) that force the execution of three processes (determining the LA's structure, distributing the tasks to perform the LA, gathering the parts of LA). The entities involved in the scenario that interact with the processes are the repository, the three consultants of the subject matter, and the LMS that automates the lecturer tasks in the preparation of a course.

Each of these processes can be observed in more detail, meaning at role level. If we decompose each process into sub-processes that assign each activity to an entity, we will obtain the roles that 


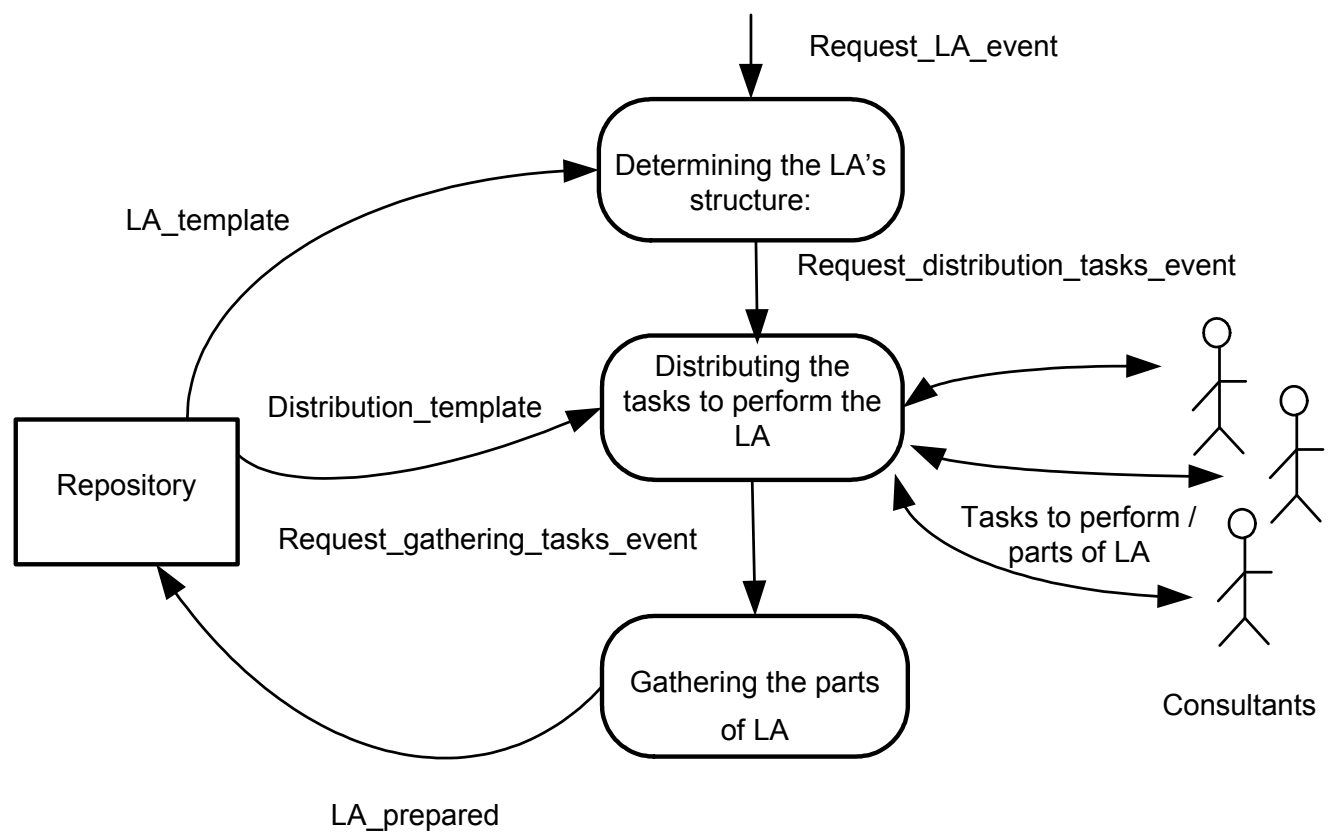

Figure 2: Diagram of the preparation of a learning activity (LA) scenario

occur to achieve the preparation of a learning activity scenario. Furthermore, each role will be able to be described by a pair of events showing the beginning and the end of the role. This level of detail lets us understand the scenario from the dynamic point of view; in terms of the flow of events.

In Table 2 the list of roles and the sequence of events required to describe the preparation of a learning activity is presented.

\section{Table 2: Detail of the roles and the corresponding flow of events in the Preparation of a Learning Activity (LA) scenario}

\begin{tabular}{|c|c|c|c|}
\hline Entity & Activity & Role & Pair of events \\
\hline $\begin{array}{l}\text { LMS/Lecturer } \\
\text { (Agent) }\end{array}$ & Receive a request & $\begin{array}{l}\text { LMS receiving the request to } \\
\text { prepare a specific LA }\end{array}$ & $\begin{array}{l}\text { Entry: reception of the initial request (preparing the } \\
\text { LA) } \\
\text { Exit: send the request to find the LA template }\end{array}$ \\
\hline $\begin{array}{l}\text { Repository } \\
\text { (Repository) }\end{array}$ & Search of LA template & $\begin{array}{l}\text { Repository searching the LA } \\
\text { template }\end{array}$ & $\begin{array}{l}\text { Entry: reception of finding the LA template request } \\
\text { Exit: delivery of the LA template }\end{array}$ \\
\hline $\begin{array}{l}\text { LMS/Lecturer } \\
\text { (Agent) }\end{array}$ & Receive a request & $\begin{array}{l}\text { LMS receiving the request to } \\
\text { distribute tasks to perform } \\
\text { the LA }\end{array}$ & $\begin{array}{l}\text { Entry: reception of distribution tasks request } \\
\text { Exit: delivery the request of searching distribution } \\
\text { tasks template }\end{array}$ \\
\hline $\begin{array}{l}\text { Repository } \\
\text { (Repository) }\end{array}$ & $\begin{array}{l}\text { Search the distribution } \\
\text { task template }\end{array}$ & $\begin{array}{l}\text { Repository searching the } \\
\text { distribution task template }\end{array}$ & $\begin{array}{l}\text { Entry: reception of finding the distribution tasks } \\
\text { template } \\
\text { Exit: delivery the distribution tasks template }\end{array}$ \\
\hline $\begin{array}{l}\text { LMS/Lecturer } \\
\text { (Agent) }\end{array}$ & Distribute tasks & $\begin{array}{l}\text { LMS determines the tasks to } \\
\text { be carried out by each con- } \\
\text { sultant }\end{array}$ & $\begin{array}{l}\text { Entry: reception of distributing tasks } \\
\text { Exit: delivery of tasks among consultants }\end{array}$ \\
\hline $\begin{array}{l}\text { LMS/Lecturer } \\
\text { (Agent) }\end{array}$ & Receive a request & $\begin{array}{l}\text { LMS receiving tasks returned } \\
\text { by each consultant }\end{array}$ & $\begin{array}{l}\text { Entry: reception of tasks from consultants } \\
\text { Exist: delivery the gathering tasks request }\end{array}$ \\
\hline $\begin{array}{l}\text { LMS/Lecturer } \\
\text { (Agent) }\end{array}$ & $\begin{array}{l}\text { Integrate tasks into the } \\
\text { LA template }\end{array}$ & $\begin{array}{l}\text { LMS integrating tasks ac- } \\
\text { cording the LA tempate }\end{array}$ & $\begin{array}{l}\text { Entry: reception of gathering tasks request } \\
\text { Exit: delivery of saving the LA request }\end{array}$ \\
\hline $\begin{array}{l}\text { Repository } \\
\text { (Agent) }\end{array}$ & Save the LA & The repository saves the LA & $\begin{array}{l}\text { Entry: reception of saving the LA } \\
\text { Exit: delivery of notification the LA created and } \\
\text { saved (the ending of the scenario) }\end{array}$ \\
\hline
\end{tabular}


It is easy to see in Table 2 that the column Role determines the activity performed by each entity at any moment. Everyone can also see that each role is constituted by two parts: the activity to be performed (column Activity) and the entity that performs it (column Entity). The OKI-OSID services can be used as entities, so the lecturer can be implemented using a common service, the agent service, and the repository can be implemented using an educational service, the repository. Furthermore, every role is activated or triggered by an entry event, and when the role in question finishes, another event, the exit event, is also activated or triggered. Hence the preparation of a learning activity scenario can be described by an expected sequence of triples (event-role-event) which makes one aware that the events that compose the flow of events have to be aligned in order to construct all the sequence of roles; the alignment of post-conditions, and pre-conditions of the roles.

\section{Conclusions and Future Work}

This paper has presented some reasons that justify the need to develop an ontology based on learning scenarios with the aim of automating the main functions of an LMS. It also sketches the knowledge domain of the proposed ontology according to the technique proposed by Noy and McGuinness (2001).

We realized that the ontology proposed has some similarities with that proposed by Amorím et al. (2006). The Amorím ontology describes the semantics of the process of designing a learning unit according to the IMS Learning design, whereas we try to describe the process by specifying learning scenarios. Both ontologies deal with similar concepts and try to describe them from a functional or dynamic perspective. In spite of everything, both ontologies have to define its main concepts establishing the ontological structure as any other ontology.

The analysis of the ontology scope has let us identify its main concepts and in consequence, it has aided determining the taxonomies required, the possible rules, and so on. For future work, we are planning to construct this ontology in Protégé and extend it using SRWL as a rule definition language. The SRWL will let us go beyond the limitations of Ontology Web Language (OWL) and so it will be useful to define the composition of learning scenarios for example.

The knowledge domain sketched has been used in the description of a specific UOC scenario using some graphical representations. In the near future, we will represent this scenario and many others using the technology previously mentioned. If we consider that one of the goals of the ontology proposed is to formalise the learning scenarios to give support to its automation, we must also be thinking of their implementation. The nature of the eLearning environment and some proposed mechanism of implementation suggest the BPEL as the suitable specification language (Bucchiarone \& Gnesi, 2006). In consequence the use of web services is mandatory and it leads us to take into account the OKI-OSID specification (Open Knowledge Initiative, 2002) to define the service interfaces. The inclusion of OKI entities in our ontology have been considered for two reasons: the abstract nature of OKI entities targeted to the higher education community and the variety of technologies that permits to interoperate through its interfaces for a given service.

All the above considerations and the specific scope of the UOC scenarios seem to lead us to the creation of several ontologies instead of only one. We propose for future work the creation of three ontologies: 1) an ontology for generic learning scenario concepts, 2) an ontology for learning scenarios in the context of the UOC University, and 3) an ontology for describing the OKIOSID elements concepts. This last ontology could possibly be split into two, if the search repository concepts are separated from the rest of the OKI-elements. 


\section{Acknowledgement}

This work has been supported by project PERSONAL - Personalizing the learning process through Adaptive Paths based on Learning Objects and Ontologies, funded by the Spanish Ministry of Education - Project code TIN2006-15107-C02-01.

\section{References}

Advanced Distributed Learning. (2004). Sharable Content Object Reference Model Version 1.3.1. Advanced Distributed Learning. Retrieved 28/01/2007 from http://www.adlnet.org

Amorim, R. R., Lama, M., Sánchez, E., Riera, A., \& Vila, X. A. (2006). A learning design ontology based on the IMS specification. Educational Technology \& Society, 9(1), 38-57.

Barros, B., Verdejo, M. F., Read, T., \& Mizoguchi, R. (2002). Applications of a collaborative learning ontology. Micai 2002: Advances in Artificial Intelligence: Second Mexican International Conference on Artificial Intelligence, Mérida, Yucatán, Mexico, 301-310.

Bucchiarone, A., \& Gnesi, S. (2006). A survey on services composition languages and models. Proceedings of International Workshop on Web Services Modelling and Testing (WS-MaTe2006), Palermo, Sicily, Italy, 51-63.

Conesa, J. (2008). Ontology driven information systems development: Pruning and refactoring of ontologies. LSI - Lenguajes y Sistemas Informáticos. Barcelona: UPC.

Gruber, T. R. (1993). A translation approach to portable ontology specifications. Knowledge Acquisition, 5(2), 199-220.

IEEE Learning Technology Standards Committee [LTSC WG12]. (2002). Draft standard for learning object metadata. Retrieved 25/10/2007 from http://ltsc.ieee.org/wg12/files/LOM_1484_12_1_v1_Final_Draft.pdf

Ikeda, M., Hoppe, U., \& Mizoguchi, R. (1995). Ontological issues of CSCL systems design. Proceedings of the $7^{\text {Th }}$ World Conference on Artificial Intelligence in Education, Washington, USA, 16-19.

IMS Global Consortium. (2003a). IMS digital repositories interoperability-core functions information model Version 1. Final Specification [IMS DRI]. Retrieved 21/10/2007 from http://www.imsglobal.org/digitalrepositories/driv1p0/imsdri infovlp0.html

IMS Global Consortium. (2003b). IMS Learning Design Information Model [IMS LD]. Version 1.0. Final Specification. Retrieved 13/07/2007 from http://www.imsglobal.org/learningdesign/ldv1p0/imsld_infov1p0.html

Inaba, A., Tamura, T., Ohkubo, R., Ikeda, M., Mizoguchi, R., \& Toyoda, J. (2001). Design and analysis of learners' interaction based on collaborative learning ontology. Proceedings of the 1st European Conference on Computer-Supported Collaborative Learning (Euro-CSCL'2001), Maastricht, The Netherlands, 308-315.

Kabel, S. C., Wielinga, B. J., \& de Hoog, R. (1999). Ontologies for indexing technical manuals for instruction. AI-ED (Artificial Intelligence in Education) Conference. Proceedings Workshop on Ontologies for Intelligent Educational Systems, Le Mans, France, 44-53.

Mizoguchi, R., Sinitsa, K., \& Ikeda, M. (1996). Task ontology design for intelligent Educational/Training systems. Positon Paper for ITS 96 Workshop on Architectures and Methods for Designing CostEffective and Reusable ITSs, Montreal, Canada, 96, 1-21.

Noy, N. F., \& McGuinness, D. L. (2001). Ontology development 101: A guide to creating your first ontology. 2001. Stanford Knowledge Systems Laboratory Technical Report KSL-01-05 and Stanford Medical Informatics Technical Report SMI-2001, 880

Open Knowledge Initiative. (2002). What is the Open Knowledge Initiative? Eduworks Corporation for OKI. 
Rius, A., Sicília, M. A., \& García-Barriocanal, E. (2008). Towards automated specifications of scenarios in enhanced learning technology. International Journal of Web-Based Learning and Teaching Technologies, 3(1), 68-78.

Rius, A., Sicília, M. A., García-Barriocanal, E., \& Macarro, G. (2006). Beyond contents and activities: Specifying processes in learning technology. Proceedings of the Fourth International Conference on Multimedia and Information and Communication Technologies in Education (M-ICTE 2006) Vol 1. Seville, Spain, 301-305.

Sánchez-Alonso, S., \& Sicilia, M. A. (2005). Normative specifications of learning objects and processes. International Journal of Instructional Technology and Distance Learning, 2(3), 3-12.

Sicilia, M. A., Garcia-Barriocanal, E., Sanchez, S., Rius, A., \& Pages, C. (2004). Specifying semantic conformance profiles in reusable learning object metadata. Proceedings of the Fifth International Conference on Information Technology Based Higher Education and Training, 2004 (ITHET 2004), Istanbul, Turkey, 93-97.

Sicilia, M. A., \& Lytras, M. D. (2005). Scenario-oriented reusable learning object characterisations. International Journal of Knowledge and Learning, 1(4), 332-341.

Simon, B., Massart, D., Van Assche, F., Ternier, S., \& Duval, E. (2005). Simple query interface specification. Draft CEN Workshop Agreement (CWA).Version, 1, 11-18.

Toffolon, C. (2006). Learning management system scenario-based engineering. Proceedings of the 5th European Conference on e-Learning (ECEL 2006), Winchester, UK, 397-406.

Tood, C., Klagholz, L, Sheschter, E., Doolan, J.Jensen, J., Nagler, I. (1999). Learning scenarios. New Jersey world languages curriculum framework. A document in support of the core curriculum content standards for world languages (pp. 75-81). New Jersey State: New Jersey State Department of Education.
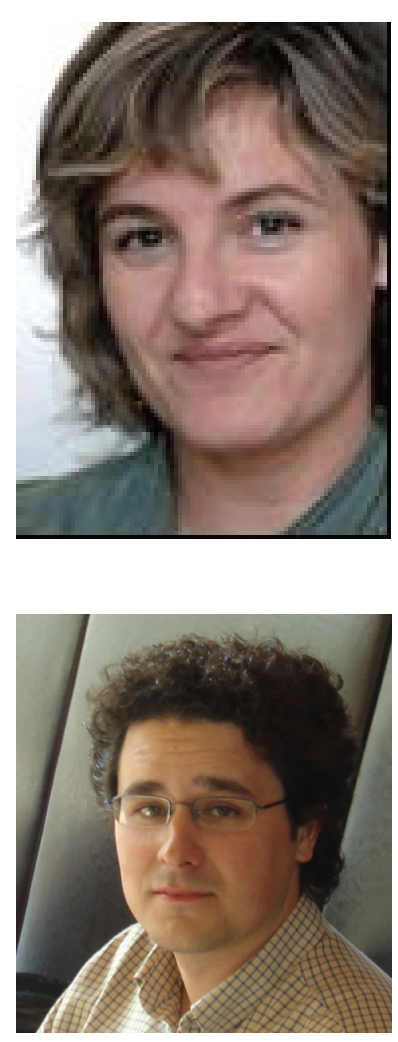

\section{Biographies}

Àngels Rius Gavídia is Assistant Professor of the Department of Computer Science, Multimedia and Telecommunication at the Open University of Catalonia, Barcelona, Spain. She is finishing her Ph.D. in the Information and Knowledge Society Program at the Open University of Catalonia. Her research interest concerns the area of e-Learning, particularly in the technological and applied aspect.

Dr. Miguel A. Sicilia is Assistant professor at the Computer Science Department of the University of Alcala (Madrid, Spain). He leads the Information Engineering Research Unit at the same university, a research group whose interests are primarily learning technology and Semantic Web, involved in several EU funded projects on these topics. $\mathrm{He}$ is also editor of several scientific journals in the field. 


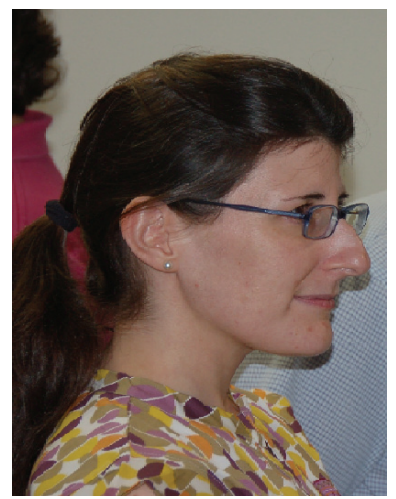

Dr. Elena García Barriocanal is assistant professor at the Computer Science Department of University of Alcalá as assistant professor. She is a member of the Information Engineering Research Unit of this University. Her research interests mainly focus on topics related to the role of knowledge representation in fields like human-computer interaction and learning technologies; concretely she actively works on ontological aspects both in e-learning and in usability and accessibility. 